\title{
Sudden death from perforation of a benign oesophageal ulcer into a major blood vessel
}

\author{
K.M. Mo, G.M. Craig, J.V. Clark and C. Champ \\ Departments of Geriatric Medicine and Pathology, Northampton General Hospital, Northampton, UK.
}

\begin{abstract}
Summary: Two cases of sudden death due to perforation of a benign oesophageal ulcer into a major blood vessel are reported. In one man, anaemia and aspiration pneumonitis dominated the clinical picture. He had an oesophageal stricture and a chronic peptic ulcer associated with an incarcerated hiatus hernia. Death was due to haemorrhage caused by perforation of the ulcer into the thoracic aorta. The second patient presented with confusion and falls, backache and indigestion. She had a hiatus hernia and a large benign chronic oesophageal ulcer. Death was due to perforation of the ulcer into the left pulmonary vein. The cases are presented for their rarity, to illustrate the complex and late presentation of problems in geriatric medicine, and as a reminder that reflux oesophagitis can be dangerous.
\end{abstract}

\section{Introduction}

We report two cases of sudden death due to perforation of a benign oesophageal ulcer into a major blood vessel. In both cases the subjects were elderly and presented with a history that was atypical and misleading.

Both cases illustrate the diagnostic difficulties that can arise in geriatric medicine, and demonstrate that reflux oesophagitis, although common, can be lethal.

\section{Case reports}

\section{Case 1}

A 72 year old male Jehovah's Witness was admitted to Northampton General Hospital in November 1986. He had not seen a doctor for 10 years but consulted his general practitioner because of a cough with yellow sputum. He gave a 12-month history of general ill health, weight loss and poor appetite, with dysphagia mainly for solids, and some backache. He admitted to indigestion and had been taking antacids bought over the counter.

On examination he was very thin, grossly anaemic and had severe dental caries. He was not short

Correspondence: G.M. Craig, M.D., M.R.C.P., St Edmund's Hospital, Northampton NN1 4DS, UK

Accepted: 24 April 1988 of breath, but there were signs of consolidation in the right middle and lower zones, and he was producing copious purulent sputum. Abdominal examination was normal, apart from bilateral reducible inguinal herniae.

Investigations showed a haemoglobin of $5.3 \mathrm{~g} / \mathrm{dl}$ and ESR of $85 \mathrm{~mm} / \mathrm{h}$. There were marked iron deficiency changes on the blood film. Two out of three samples for faecal occult blood were negative. His chest X-ray showed a large opacity at the right base, suggestive of an underlying neoplasm. There were some inflammatory changes and several old rib fractures were noted. Lumbar spine X-rays showed collapse of T12, L2, 3 and 4. Diagnostic pleural tap produced blood stained fluid containing no malignant cells. Sputum cytology was negative. Sputum culture grew no pathogens. Tests for myeloma were negative.

$\mathrm{He}$ refused transfusion for religious reasons. $\mathrm{He}$ received erythromycin and flucloxacillin for his pneumonia without response. A repeat chest X-ray after 2 weeks of antibiotic treatment showed two air fluid levels apparently in the right basal opacity, but there was some doubt as to whether the changes represented a pleural loculus or a lung abscess. In view of his dental caries a lung abscess seemed likely and oral metronidazole was added to his regime with clinical and radiological improvement.

In view of his iron deficiency anaemia and

(C) The Fellowship of Postgraduate Medicine, 1988 
dysphagia a barium swallow and meal was performed. This showed a tortuous oesophagus with little peristaltic activity but with tertiary contractions. No intrinsic mass or stricture was identified and the stomach was normal. $\mathrm{He}$ refused endoscopy.

One month after admission he had a small haematemesis and became anxious and paranoid, requiring mild sedation. He was started on ranitidine. His general condition improved and his haemoglobin rose to $7.7 \mathrm{~g} / \mathrm{dl}$ but his ESR remained high. A chest X-ray 10 weeks after admission showed marked clearing on the right, with some residual inflammatory change in the mid-zone and some pleural thickening at the right base. There was a new area of consolidation in the left midzone. Five weeks after his first small haematemesis he had a massive haematemesis and died.

At post mortem the lower two-thirds of the oesophagus was found to be greatly dilated. There was a stricture just above the gastro-oesophageal junction, where there was a chronic peptic ulcer which had penetrated through the oesophagus into the adjacent thoracic aorta, the wall of which bore a $3 \mathrm{~mm}$ diameter perforation. The stomach contained a large volume of blood and there was a hiatus hernia, the proximal $30-40 \mathrm{~mm}$ of stomach being incarcerated in the mediastinum. The lungs showed very dense pleural adhesions, particularly over the right lower lobe, and the cut surface of both lower lobes contained patchy areas of consolidation, compatible with previous episodes of aspiration pneumonitis produced by the oesophageal stricture.

\section{Case 2}

An 82 year old female was admitted to Danetre Hospital, Daventry in September 1986 following a fall at home. There was a history of bouts of confusion and fever over the past 3-4 days, with one or two falls.

We obtained a history of months or years of backache felt in the thoracic region, and of indigestion which sometimes kept her awake at night. She had been living on a proprietary antacid (Rennies). Mild iron deficiency anaemia had been noted in the past and she was on oral iron.

On examination, she had bruising over the spine at C8 and T2 posteriorly, and on her left shoulder. The left leg was short and externally rotated, but she could move the hip freely. Abdominal examination was normal.

Preliminary investigations showed a haemoglobin of $11.1 \mathrm{~g} / \mathrm{dl}$. On the day following admission she was restless, had a small haematemesis, then a few hours later suddenly became cyanosed and died. As she was turned after death a large volume of blood regurgitated into the mouth. At post mortem the oesophagus was found to contain a large chronic ulcer on the anterior wall. The ulcer commenced $10 \mathrm{~mm}$ below the carina and measured $40 \mathrm{~mm}$ in length. The lower border of the ulcer almost reached the oesophagogastric junction, the stomach lying in the chest through a hiatus hernia. At a point $25 \mathrm{~mm}$ below the carina, the deeply penetrating benign ulcer had ulcerated into the left pulmonary vein, through a defect $3 \mathrm{~mm}$ in diameter. The stomach contained blood.

\section{Discussion}

An aorto-oesophageal fistula secondary to reflux oesophagitis is extremely rare. Cronen ${ }^{1}$ reported two cases and traced three other published cases. In three out of five the diagnosis, as in our case, was made only at post mortem. Cronen emphasized that severe arterial bleeding in a patient with reflux oesophagitis should alert one to the possibility of an aorto-oesophageal fistula. ${ }^{1}$

The great majority of aorto-oesophageal fistulae are associated with a thoracic aortic aneurysm, ${ }^{2,3}$ although cases due to oesophageal erosion by a foreign body ${ }^{4}$ and miscellaneous pathology, includ ing carcinoma of the oesophagus, can occur.

Chiari ${ }^{5}$ described a triad of symptoms and signs? associated with an aorto-oesophageal fistula, namely mid-thoracic pain, a warning or sentinel mild haematemesis, and, after a symptom-free interval, a massive upper gastrointestinal haemorrhage. In $80 \%$ of cases the oesophageal perforation develops gradually over several days or weeks ${ }^{6}$ with bleeding initially being mild and intermittent. ${ }^{7}$ These features refer however to the erosion of thoracic aortic aneurysms into the oesophagus a different cause of aorto-oesophageal fistulae from those in our patients.

It is recognized that oesophageal radiology rarely demonstrates leakage of contrast material into the aorta in cases of aorto-oesophageal fistula. It has been suggested that an oral oesophagogram should include air contrast and rotational views to avoid missing an early lesion. ${ }^{7}$ Others consider aortography to be the most definitive diagnostic procedure for aorto-oesophageal fistulae. ${ }^{3}$ Cronen suggested that thoracic aortography should be performed if coeliac arteriography does not demonstrate the bleeding site, but a bleeding rate of 0.5 to $1 \mathrm{ml} / \mathrm{min}$ may be required to demonstrate the fistula. ${ }^{1}$

The diagnosis of aorto-oesophageal fistula was not made in our first patient because our barium studies were misleading, and recurrent aspiration pneumonia dominated the clinical picture. 
A pulmonary venous oesophageal fistula due to oesophageal reflux (Case 2) must be excessively rare, and our case may be unique. Isolated cases of perforation of a gastric ulcer in a hiatus hernia into the left atrium, ${ }^{8}$ right atrium ${ }^{9}$ and left ventricle ${ }^{10}$ have been reported. We have been told of a case of perforation of an ulcer into a coronary artery causing angina as a presenting complaint, but the Northampton hospital records cannot be traced

\section{References}

1. Cronen, P., Snow, N. \& Nightingale, D. Aortoesophageal fistula secondary to reflux esophagitis. Ann Thorac Surg 1982, 33: 78-80.

2. Carter, R., Mulder, G.A., Snyder, E.N. \& Brewer, L.A. Aortoesophageal fistula. Am J Surg 1987, 136: 26-30.

3. Han, S.Y., Jander, H.P. \& Ho, K.J. Aortoesophageal fistula. South Med J 1981, 74: 1260-1262.

4. El Barbary, A.S., Foad, H. \& Fathi, A. Oesophageal fistulae caused by swallowed foreign bodies. J Laryngol Otol 1969, 83: 251-259.

5. Chiari, H. Ueber Fremdköperverletzung des Oesophagus mit Aortenperforation. Berl Klin Wochenschr 1914, 51: 7.

6. Sinar, D.R., Demaria, A., Kataria, Y.P. \& Thomas, F.B. Aortic aneurysm eroding the esophagus, case report and review. Am J Dig Dis 1977, 22: 252-254.
(Graham and Samtani, personal communication).

These two cases are presented for their rarity, to illustrate the complex and late presentation of problems in geriatric medicine, and as a reminder that reflux oesophagitis can be dangerous. Many elderly patients present with iron deficiency anaemia associated with a hiatus hernia and reflux oesophagitis. We should all be more alert to the potentially lethal complications that may develop.

7. Myers, H.G. \& Silber, W. Oesophageal bleeding from aorto-oesophageal fistula due to aortic aneurysm. $S$ Afr Med J 1983, 63: 124-127.

8. Scott, A., Garland, C. \& Gillespie, G. Peptic ulceration of the left atrium. $J R$ Coll Surg Edinb 1977, 22 (5): 331-332.

9. Frey, E., Perforation of a gastric ulcer from a hiatus hernia into the pericardium. Fortschr Roentgenstr 1961, 95: 852-855.

10. Kissel, P., Schmitt, J., Barrucand, D., Vaillant, G. \& Reny, J. Hemmorragie digestive secondair a la perforation d'un ulcere gastrique dans le ventricule gauche (a propos d'une observation anatomo-clinique). Ann Med Nancy 1964, 3: 1022-1028. 